\title{
Computação Aplicada na Assistência Educacional em Doenças Crônicas Não Transmissíveis: um Mapeamento Sistemático
}

\author{
Andrêsa Vargas Larentis ${ }^{1}$, Débora Nice Ferrari Barbosa ${ }^{2}$, Carla Rosana da Silva ${ }^{3}$, \\ Jorge Luis Victória Barbosa ${ }^{1}$ \\ ${ }^{1}$ Programa de Pós-Graduação em Computação Aplicada (PPGCA) \\ Universidade do Vale do Rio dos Sinos (UNISINOS) \\ ${ }^{2}$ Programa de Pós-Graduação em Diversidade Cultural e Inclusão Social \\ Universidade Feevale \\ ${ }^{3}$ Associação de Assistência em Oncopediatria (AMO Criança) \\ andresa.vargas@gmail.com, deboranice@feevale.br, \\ projetoseamocrianca.com.br, jbarbosa@unisinos.br
}

\begin{abstract}
The purpose of this research is to identify studies that applied computing in the assistance of noncommunicable diseases (NCDs). The text presents the results of a systematic mapping study that used eight different data sources known in the academic field regarding both data science and health science, including papers published between 2008 and 2018. The search resulted in 20819 papers and 51 were selected for analysis. The results characterize the use of computing in the promotion of education in NCDs. Diabetes was the chronic disease prioritized (60.78\%). About 49.02\% of the solutions used data from the individuals to assist in their health conditions. In addition, $58.82 \%$ produced outcome measures by means of experiments.
\end{abstract}

Resumo. O objetivo desta pesquisa é identificar estudos que apliquem a computação na assistência em doenças crônicas não transmissíveis (DCNTs). $O$ texto apresenta os resultados de um mapeamento sistemático realizado em oito bases de dados conhecidas no âmbito acadêmico nas áreas de ciência da computação e ciências da saúde incluindo publicações entre 2008 e 2018. A busca resultou em 20819 artigos e 51 foram selecionados para análise. Os resultados caracterizam o uso da computação na promoção da educação em DCNTs. Diabetes foi a doença crônica priorizada (60,78\%). Cerca de 49,02\% das soluções usaram dados dos indivíduos no auxílio as condições de saúde. Além disso, 58,82\% produziram resultados mensuráveis por experimentos.

\section{Introdução}

Segundo dados da World Health Organization (WHO), no ano de 2016 morreram em torno de 41 milhões de pessoas devido a doenças crônicas não transmissíveis (DCNTs), o equivalente a $71 \%$ de um total de 57 milhões de pessoas que morreram no mundo. A maioria destas mortes foi causada por quatro principais doenças crônicas: doenças cardiovasculares representaram 17,9 milhões de mortes, cânceres 9 milhões, doenças crônicas pulmonares 3,8 milhões e diabetes 1,6 milhões [WHO 2018]. 
VIII Congresso Brasileiro de Informática na Educação (CBIE 2019)

Anais do XXX Simpósio Brasileiro de Informática na Educação (SBIE 2019)

O diagnóstico das DCNTs provoca um forte impacto na sociedade em geral. Hábitos como sedentarismo e tabagismo contribuem para o aumento de diagnósticos. Por sua vez a hipertensão arterial, o sobrepeso e a obesidade, resultam em alterações metabólicas e fisiológicas no organismo do indivíduo e compõem os principais fatores de risco que devem ser controlados como forma de prevenir casos destas doenças [WHO 2013]. O controle dos fatores de risco, mediado através de um processo educacional, pode ser utilizado como forma de prevenir o diagnóstico das DCNTs.

As tecnologias digitais possibilitam o acesso à informação através de diferentes recursos digitais em qualquer local e tempo. Neste contexto, a computação ubíqua surge como um modelo computacional que está sempre presente para atender às necessidades do usuário, de forma natural e imperceptível [Satyanarayanan 2001]. Esta abordagem é aplicável em áreas, tais como educação [Heidrich et al. 2018], saúde [Vianna e Barbosa 2019] e bem-estar [Vianna et al. 2017].

$\mathrm{Na}$ área da educação voltada para a saúde, desenvolver sistemas educacionais para apoiar atividades de aprendizagem relacionadas com computação ubíqua tem sido de interesse de pesquisas [Quinde et al. 2018 e Seys et al. 2018]. A assistência médica ao indivíduo vai além dos consultórios e hospitais. Locais públicos e o lar do paciente também são espaços onde serviços de saúde e educação podem ser ofertados. Por sua vez, a educação ubíqua [Cárdenas-Robledo e Peña-Ayala 2018] tem se mostrado como uma possibilidade no desenvolvimento de sistemas educacionais que suportem atividades de aprendizagem onipresentes, levando-se em consideração o contexto do indivíduo.

Nesse sentido, o artigo apresenta um mapeamento sistemático da literatura para identificar estudos que envolvam a aplicação da computação na educação para assistência em DCNTs. A motivação deste trabalho é conhecer as tecnologias, técnicas, doenças crônicas e resultados, de modo a construir uma referência sobre o tema para pesquisas futuras. Além disso, espera-se identificar estudos que envolvam a computação ubíqua, e de forma mais específica a educação ubíqua [Cárdenas-Robledo e Peña-Ayala 2018], tema considerado estratégico na assistência em DCNTs. Este artigo está organizado em quatro seções. A seção 2 descreve a metodologia utilizada no mapeamento sistemático. Na seção 3 são apresentados e discutidos os resultados. Finalmente, a seção 4 aborda as considerações finais.

\section{Metodologia do Mapeamento Sistemático}

O mapeamento sistemático utiliza a metodologia proposta por Petersen et al. (2008, 2015), cuja execução envolve os seguintes passos: 1) Definir as questões de pesquisa; 2) Definir o processo de pesquisa; 3) Definir os critérios para seleção dos artigos e

4) Executar as análises e classificar os artigos selecionados.

\subsection{Questões de pesquisa}

Esse trabalho responde as seguintes questões de pesquisa: 1) Como a computação pode promover a assistência educacional em DCNTs? 2) Quais tecnologias estão sendo utilizadas para apoiar a assistência educacional em DCNTs? 3) Quais são as DCNTs priorizadas na assistência educacional dos sistemas computacionais? 4) Quais estratégias de avaliação estão sendo usadas para medir os resultados de experimentos com pessoas? 5) Existem sistemas computacionais que aplicam a educação ubíqua no 
VIII Congresso Brasileiro de Informática na Educação (CBIE 2019)

Anais do XXX Simpósio Brasileiro de Informática na Educação (SBIE 2019)

processo de aprendizagem? 6) Existem sistemas computacionais adaptáveis às necessidades do indivíduo (personalização)? 7) Qual a distribuição dos artigos por base de dados e o local de publicação a cada ano?

\subsection{Processo de pesquisa}

O processo de pesquisa consistiu em: 1) Especificar a string de busca; 2) Escolher as bases de dados para aplicar a string e 3) Executar a string e obter os resultados.

A string de busca foi definida como: (("system" OR "application" OR "computational model" OR "architecture" OR "platform" OR "pervasive" OR "mobile" OR "ubiquitous") AND ("learning" OR "education") AND ("noncommunicable diseases" OR "chronic diseases" OR "risk factors" OR "chronic conditions" OR "diabetes" OR "cancers" OR "cardiovascular diseases" OR "respiratory diseases")).

Posteriormente, foram definidas oito bases de dados para execução da string de busca. Além disso, definiu-se os campos "Abstract", "Title" e "Keyword" para a busca. As customizações de cada uma das bases de dados são apresentadas na Tabela 1.

Tabela 1. Customizações da string de busca nas bases de dados.

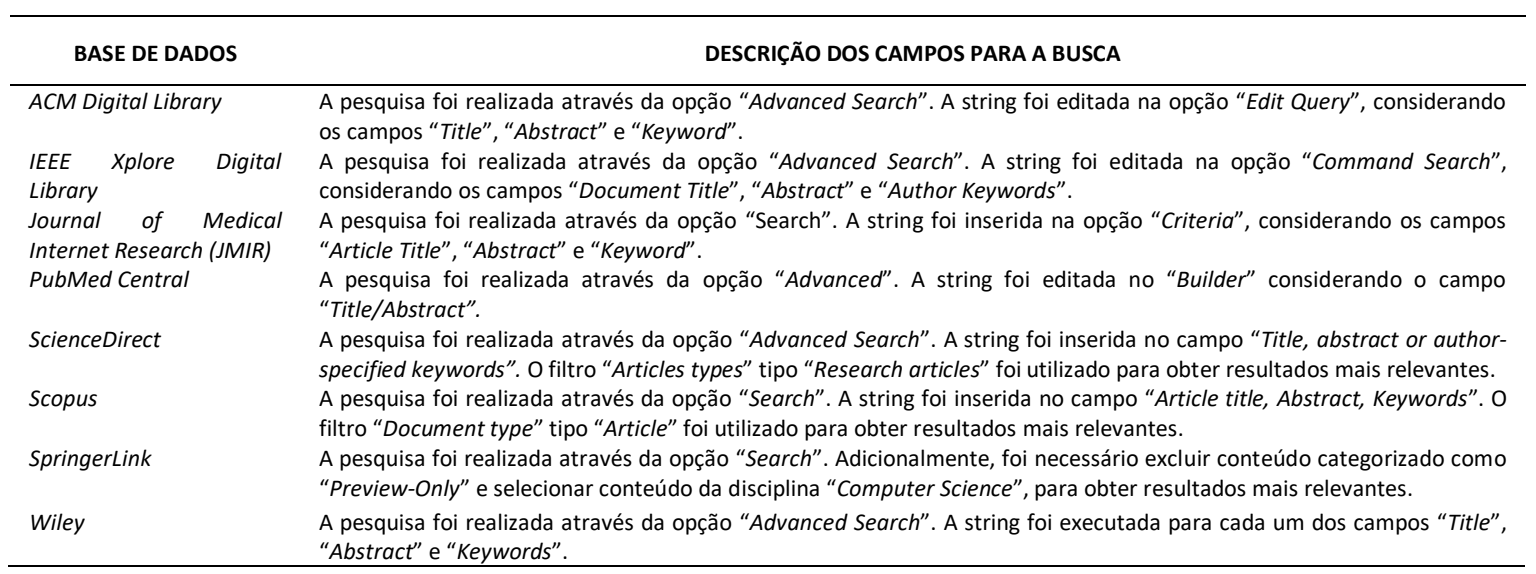

\subsection{Critérios de inclusão e exclusão}

Os critérios de inclusão (CI) definidos foram: 1) o artigo deve estar publicado em uma conferência, workshop ou periódico; 2) o artigo deve estar relacionado ao uso da educação na assistência em DCNTs e 3) o artigo deve ser um texto original publicado integralmente. Além disso, foram considerados os seguintes critérios de exclusão (CE): 1) artigos publicados antes de $2008 ; 2$ ) artigos que não estão escritos em inglês; 3) textos que estão publicados em livros, dissertações ou teses; 4) artigos não relacionados com as questões de pesquisa; 5) artigos relacionados com educação, mas não com a área da saúde e 6) artigos duplicados.

\section{Resultado e discussões}

A Figura 1 resume as etapas do processo de pesquisa e apresenta o total de artigos resultantes em cada etapa por base de dados. Após a execução da string de busca foram encontrados 20819 artigos. Ao término da aplicação dos critérios CE1, CE2 e CE3 foram selecionados 11156. O próximo passo consistiu na verificação por título e palavras-chave aplicando-se o CE4, resultando em 408. Em seguida, os textos foram analisados por resumo aplicando-se o CE5, resultando em 150. Foram identificados 19 
VIII Congresso Brasileiro de Informática na Educação (CBIE 2019)

Anais do XXX Simpósio Brasileiro de Informática na Educação (SBIE 2019)

em duplicidade aplicando-se o CE6, os quais foram removidos, resultando em 131. O artigo de Mendes Neto et al. (2014) foi adicionado devido a sua relevância para a pesquisa, totalizando 132 artigos. O artigo foi selecionado na base de dados IEEE Xplore Digital Library e apesar de ter o título em inglês foi publicado em português. $\mathrm{O}$ artigo explora a assistência educacional na saúde e cita a educação ubíqua.

O próximo filtro seguiu os passos da "Three-pass approach" introduzida por Keshav (2007). O primeiro passo consiste em: a) leitura do título, resumo e introdução; b) leitura do título das seções e subseções; c) verificação do conteúdo matemático (caso aplicável) e d) leitura das conclusões. O segundo consiste em analisar as figuras, diagramas e outras ilustrações no texto. Finalmente, o terceiro passo requer uma leitura de todo o texto. Os 132 artigos remanescentes foram analisados, sendo 51 selecionados para responder as questões de pesquisa, além de servirem como base para discussões sobre o tema abordado nesse trabalho.

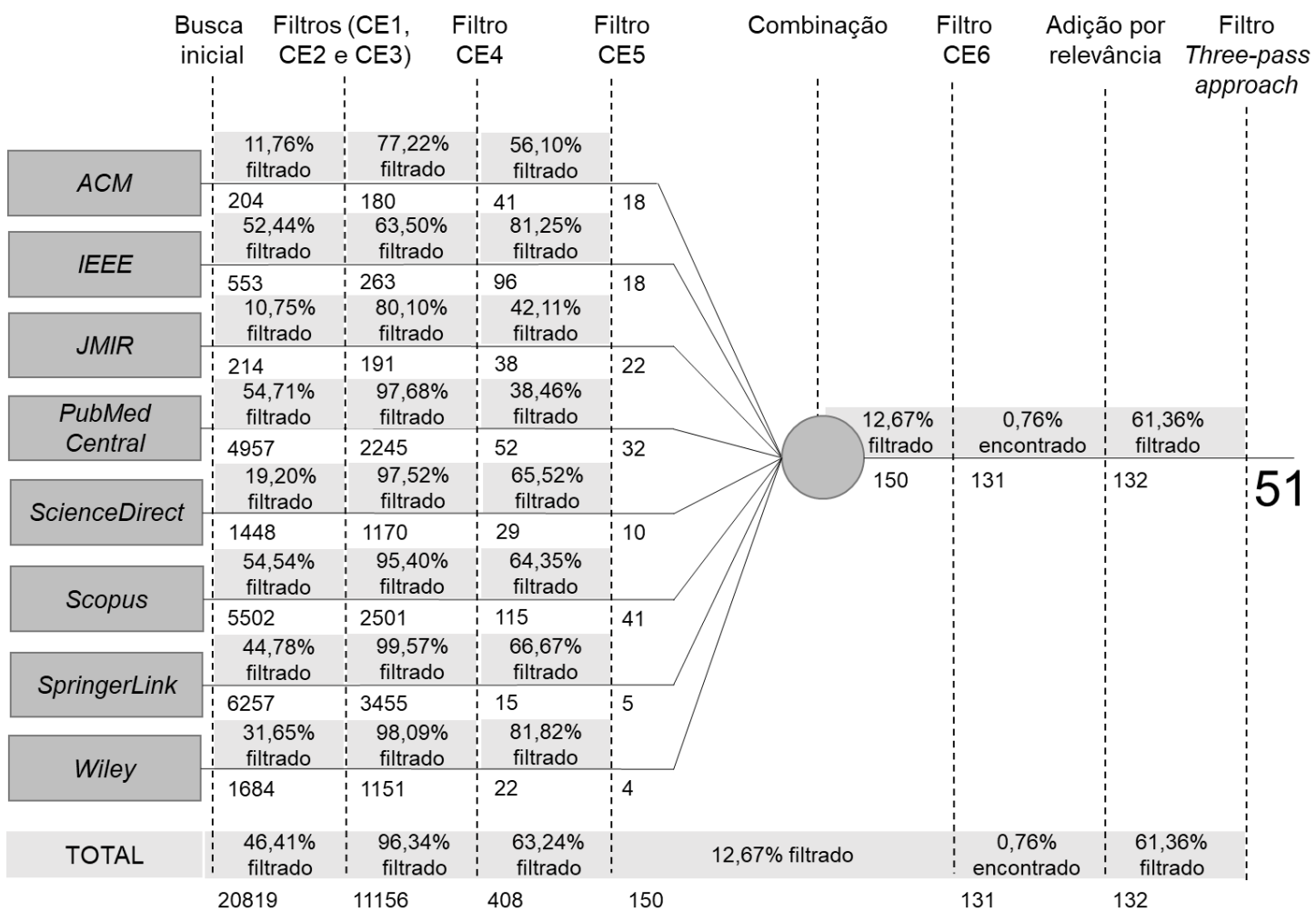

Figura 1. Etapas do processo de pesquisa.

A Tabela 2 apresenta os 51 artigos organizados por identificação (ID), autores, título, conferência, periódico ou workshop onde foi publicado e ano de publicação. Visando responder a primeira questão "Como a computação pode promover a assistência educacional em DCNTs?”, os artigos foram classificados em seis categorias de acordo com as funcionalidades disponíveis na solução para o uso da computação na promoção da educação: Educational Content (20 artigos, IDs = 2, 5, 6, 7, 8, 10, 13, 14, 23, 27, 29, 30, 31, 33, 38, 39, 40, 44, 46, 50), Self-management and Educational Content $(21$, IDs $=1,3,9,15,16,19,20,21,22,24,26,28,34,37,41,42,43,45,47,48,51)$, Self-management of Diabetes and Games (1, ID = 49), Virtual Community (1, ID = 17), Games (6, IDs = 4, 11, 18, 25, 35, 36), Custom Content (2, IDs = 12, 32). 
VIII Congresso Brasileiro de Informática na Educação (CBIE 2019)

Anais do XXX Simpósio Brasileiro de Informática na Educação (SBIE 2019)

Tabela 2. Lista dos artigos selecionados.

\begin{tabular}{cl}
\hline ID & \multicolumn{1}{c}{ AUTORES/TítUlo/PUBLICAÇÃo/ANO } \\
\hline 1 & [Kumar et al.] A Diabetes Mobile App With In-App Coaching From a Certified Diabetes Educator Reduces A1C for Individuals With Type 2 \\
Diabetes. (The Diabetes Educator) 2018 & \\
& [Alotaibi] A mobile Diabetes educational system for fasting Type-2 diabetics in Saudi Arabia. (2nd International Conference on Information
\end{tabular}

Technology, Computer and Electrical Engineering) 2015

3 [Alotaibi and Albalawi] A Mobile Gestational Diabetes Management and Educational System for Gulf Countries: System Architecture. (9th IEEE Control and System Graduate Research Colloquium) 2018

[Bomark et al.] A prototype social learning platform for children with diabetes type 1. (16th International Academic MindTrek Conference) 2012

[Kerfoot et al.] A Team-Based Online Game Improves Blood Glucose Control in Veterans With Type 2 Diabetes: A Randomized Controlled Trial. (Diabetes Care) 2017

6 [Diefenbach et al.] Acceptability and preliminary feasibility of an Internet/CD-ROM-based education and decision program for early-stage prostate cancer patients: Randomized pilot study. (Journal of Medical Internet Research) 2012

7 [Janssen et al.] An Online Learning Module to Increase Self-Efficacy and Involvement in Care for Patients With Advanced Lung Cancer: Research Protocol. (JMIR Research Protocols) 2016

8 [Tani et al.] An online support system for stroke education - Focusing on use of the system by schoolteachers in junior high schools. (7th International Symposium on Medical Information and Communication Technology) 2013

[Stokes et al.] Chronic Care Continuum (C3): Mobile-ready Life Skills Training for Adolescents with Chronic Diseases. (First ACM Workshop on Mobile Systems, Applications, and Services for Healthcare) 2011

10 [Sirotinin and George] Computer-aided learning in insulin pump training. (Journal of Diabetes Science and Technology) 2010

11 [Mass et al.] Concept Development of the Eindhoven Diabetes Education Simulator Project. (Games for Health Journal) 2016

12 [Mendes Neto et al.] Content's personalized recommendation for implementing ubiquitous learning in health 2.0. (IEEE Latin America Transactions) 2014

[Mendu et al.] Design of a Culturally-Informed Virtual Human for Educating Hispanic Women About Cervical Cancer. (12th EAl International Conference on Pervasive Computing Technologies for Healthcare) 2018

14 [Guo] Design of mobile e-books as a teaching tool for diabetes education. (13th International Conference Mobile Learning) 2017

15 [Alvarado-Martel et al.] Design, construction, and implementation of an online platform for patients with type 1 diabetes: EncoDiab. (Patient Preference and Adherence) 2015

16 [Winterlich et al.] Diabetes Digital Coach: Developing an Infrastructure for e-Health Self-Management Tools. (9th International Conference on Developments in eSystems Engineering) 2016

17 [Vorderstrasse et al.] Diabetes LIVE (Learning in Virtual Environments): Testing the Efficacy of Self-Management Training and Support in 7 Virtual Environments (RCT Protocol). (Nursing Research) 2015

18 [Baghaei et al.] Diabetic Mario: Designing and Evaluating Mobile Games for Diabetes Education. (Games for Health Journal) 2016

[Boels et al.] Effectiveness of diabetes self-management education via a smartphone application in insulin treated type 2 diabetes patients design of a randomised controlled trial ('TRIGGER study'). (BMC Endocrine Disorders) 2018

20 [Gustin et al.] Empowerment of diabetic patients through mHealth technologies and education: development of a pilot self-management application. (13th International Conference on Medical Information Processing and Analysis) 2017

21 [Baek et al.] Enhancing user experience through user study: Design of an mhealth tool for self-management and care engagement of cardiovascular disease patients. (JMIR Cardio) 2018

[Wonggom et al.] Evaluation of the effectiveness of an interactive avatar-based education application for improving heart failure patients'

22 knowledge and self-care behaviours: A pragmatic randomized controlled trial protocol. (Journal of Advanced Nursing) 2018

[Hussain and Athula] Extending a conventional chatbot knowledge base to external knowledge source and introducing user based sessions for diabetes education. (32nd International Conference on Advanced Information Networking and Applications Workshops) 2018

24 [Sultan and Mohan] Facilitating Informal Learning in a Mobile Application for a Group of Type 2 Diabetics. (11th International Conference on Advanced Learning Technologies) 2011

[Pesare et al.] Game-based learning and Gamification to promote engagement and motivation in medical learning contexts. (Smart Learning Environments) 2016

26 [Hidalgo et al.] GIUCModel: A monitoring and modeling system for chronic diseases applied to diabetes. (Journal of Biomedical Informatics) 2014

27 [Jiménez et al.] Heuristic Evaluation of a Gamified Application for Education in Patients with Diabetes. (7th Mexican Conference on HumanComputer Interaction) 2018

28 [Guo et al.] Impact of Mobile Diabetes Self-Care System on patients' knowledge, behavior and efficacy. (Computers in Industry) 2015

[Almotairi et al.] Improving Patient Outcomes through Customized Learning. (32nd International Conference on Advanced Information Networking and Applications Workshops) 2018

[Partridge et al.] Innovating structured education for people with type 1 diabetes: Www.Bertieonline.org.UK. (Journal of Diabetes Nursing) 2017

31 [Pulman et al.] Innovative mobile technology alcohol education for young people with type 1 diabetes. (Practical Diabetes) 2013

[Gray et al.] Internet protocol television for personalized home-based health information: Design-based research on a diabetes education system. (JMIR Research Protocols) 2014

33 [Finkelstein et al.] Introducing a Blackberry eLearning platform for interactive hypertension education. (2nd International Conference on Mobile, Hybrid, and On-Line Learning) 2010

[Akker et al.] Let Them Play: Experiences in the Wild with a Gamification and Coaching System for Young Diabetes Patients. (11th EA International Conference on Pervasive Computing Technologies for Healthcare) 2017

35 [Orji et al.] LunchTime: a slow-casual game for long-term dietary behavior change. (Personal and Ubiquitous Computing) 2013

[Glasemann et al.] Making Chocolate-covered Broccoli: Designing a Mobile Learning Game about Food for Young People with Diabetes. (8th ACM Conference on Designing Interactive Systems) 2010

37 [Bickmore et al.] Managing Chronic Conditions with a Smartphone-based Conversational Virtual Agent. (18th International Conference on Intelligent Virtual Agents) 2018

[Praditsangthong and Bhattarakosol] M-Learning System: A mobile learning for asthma. (12th International Conference on Electrical Engineering/Electronics, Computer, Telecommunications and Information Technology) 2015

[Finkelstein and Wood] Mobile eLearning platform for interactive patient education. (International Conference on Mobile, Hybrid, and On-line Learning) 2009

40 [Boyd et al.] My Interventional Drug-Eluting Stent Educational App (MyIDEA): Patient-Centered Design Methodology. (JMIR Mhealth Uhealth) 
VIII Congresso Brasileiro de Informática na Educação (CBIE 2019)

Anais do XXX Simpósio Brasileiro de Informática na Educação (SBIE 2019)

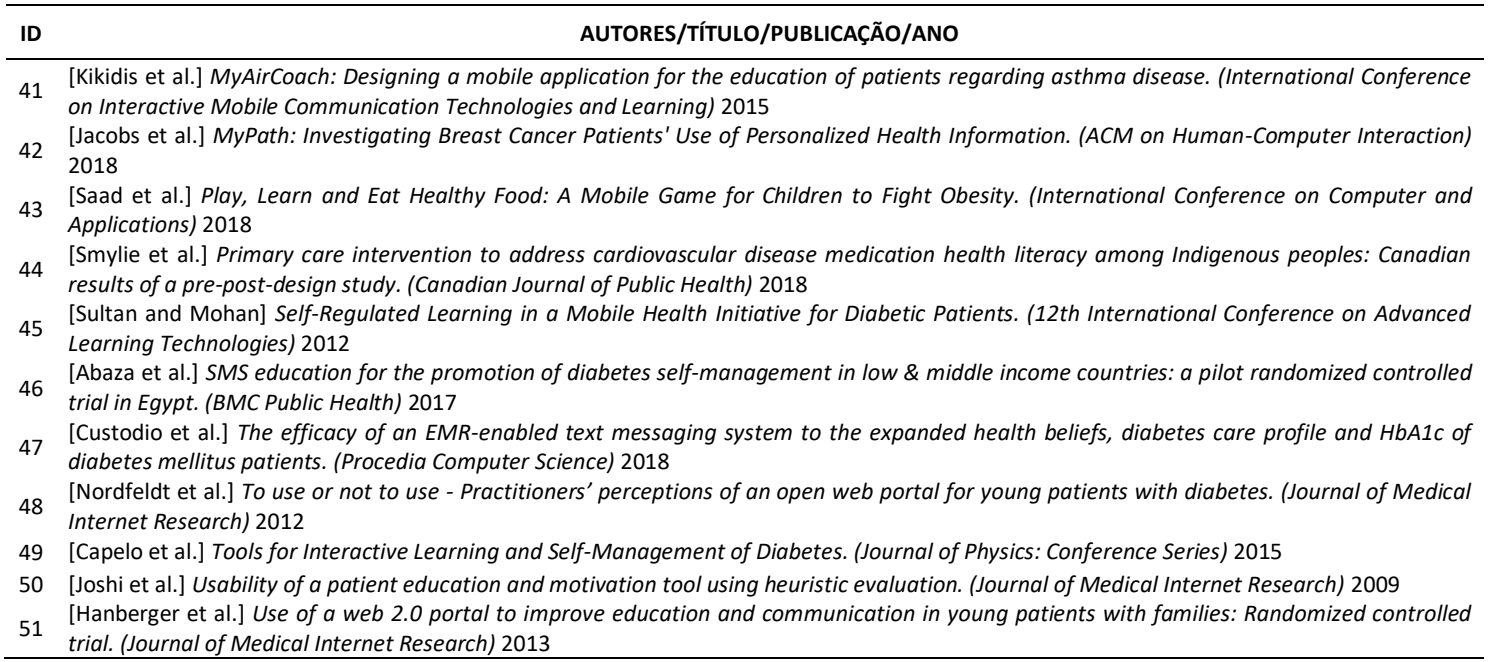

Em relação a segunda questão "Quais tecnologias estão sendo utilizadas para apoiar a assistência educacional em DCNTs?", os artigos foram classificados em sete categorias de acordo com a abordagem tecnológica utilizada no desenvolvimento da solução para apoiar a educação: Computer Application (2 artigos, IDs = 6, 10), Internet Protocol Television (IPTV) (1, ID = 32), Mobile Application (25, IDs = 1, 5, 9, 12, 13, 14, 19, 20, 21, 22, 24, 25, 27, 28, 31, 33, 36, 37, 39, 40, 41, 42, 45, 46, 47), Mobile Application e Web Application (8, IDs = 2, 3, 4, 16, 34, 35, 38, 43), Mobile Games (2, IDs $=11,18)$, Virtual Environments $(1, \mathrm{ID}=17)$ e Web Application $(12$, IDs = 7, 8, 15, $23,26,29,30,44,48,49,50,51$ ).

A terceira questão “Quais são as DCNTs priorizadas na assistência educacional dos sistemas computacionais?" refere-se ao tipo de doença crônica mencionada, sendo assim os artigos foram classificados em: Asthma (3 artigos, IDs = 38, 39, 41), Transient Ischemic Attack (1, ID = 8), Prostate Cancer (1, ID = 6), Lung Cancer (1, ID = 7), Diabetes (19, IDs = 3, 10, 11, 14, 16, 18, 23, 26, 27, 28, 32, 35, 36, 45, 46, 47, 48, 49, 51), Type 1 Diabetes (6, ID = 4, 15, 20, 30, 31, 34), Type 2 Diabetes (6, IDs = 1, 2, 5, 17, 19, 24), Cardiovascular Disease (4, IDs $=21,22,40,44)$, Chronic Diseases (6, IDs $=9,12,25,29,37,50)$, Hypertension (1, ID = 33), Cervical Cancer (1, ID = 13), Breast Cancer (1, ID = 42) e Obesity (1, ID =43). A Figura 2 apresenta a distribuição dos percentuais de cada uma das categorias. O percentual de artigos relacionados aos três tipos de diabetes é de $60,78 \%$, ou seja, 31 em um total de 51 .

Quanto a quarta questão "Quais estratégias de avaliação estão sendo usadas para medir os resultados de experimentos com pessoas?", 30 artigos (o equivalente a $58,82 \%$ ) produziram resultados através de experimentos com pacientes, profissionais de saúde ou pessoas em geral, sendo estes classificados em Randomized Controlled Trials (RCT) (8 artigos, IDs = 5, 19, 22, 37, 40, 46, 47, 51), Randomized Pilot Study (RPS) (1, ID = 6), Pilot Study (PS) (20, IDs = 1, 8, 10, 12, 13, 18, 21, 23, 25, 27, 28, 32, 33, 34, 35, 42, 43, 45, 48, 50) e Pre-post-design Study (PDS) (1, ID = 44). Os demais não fizeram menção a resultados de experimentos conduzidos com pessoas, concentrando-se no processo de desenvolvimento do sistema e na apresentação das suas funcionalidades. 
VIII Congresso Brasileiro de Informática na Educação (CBIE 2019)

Anais do XXX Simpósio Brasileiro de Informática na Educação (SBIE 2019)

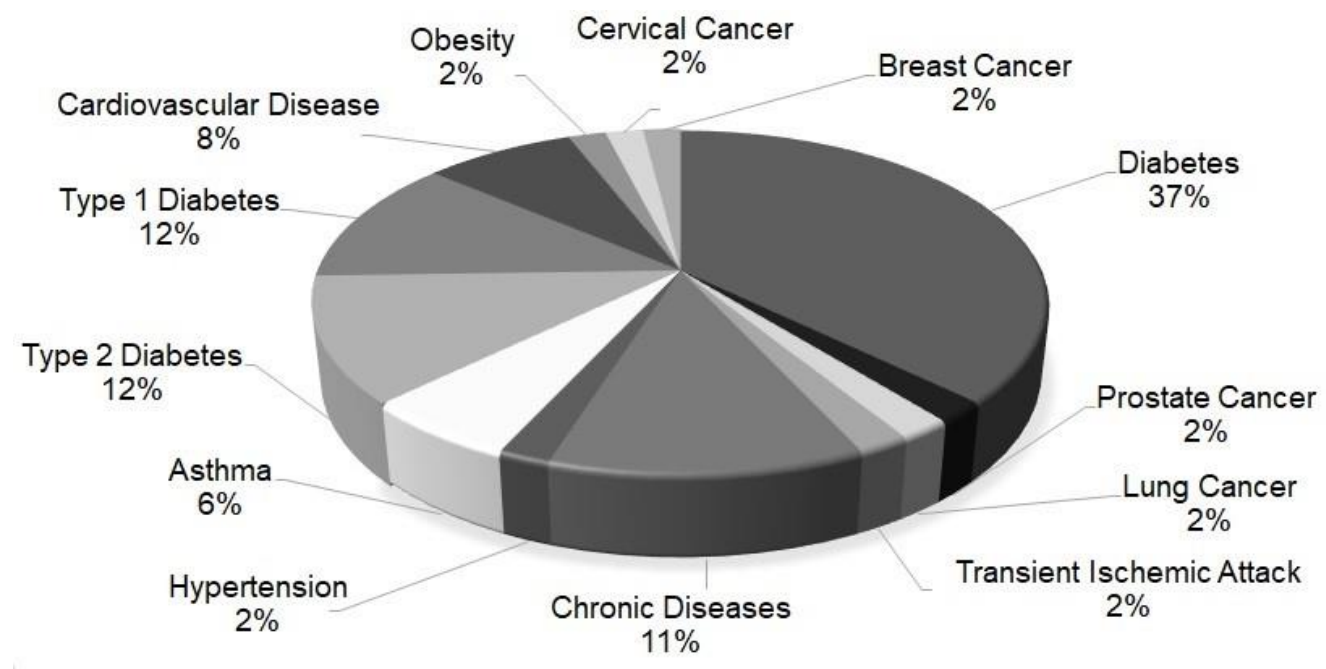

Figura 2. Total de artigos por doença crônica.

A Tabela 3 sumariza as principais características dos artigos que apresentaram resultados de experimentos envolvendo pessoas.

Tabela 3. Características dos experimentos com resultado mensurável.

\begin{tabular}{|c|c|c|c|c|}
\hline Autores e IDs na Tabela 2 & Estratégia & Participantes & Qtde & Resultado mensurável \\
\hline Tani et al. [8] & PS & Alunos e professores & 78 & Conhecimento sobre acidente vascular cerebral \\
\hline Sirotinin and George [10] & PS & Profissionais da saúde & 126 & Conhecimento dos profissionais sobre saúde \\
\hline Mendes Neto et al. [12] & PS & Pacientes diabéticos & 10 & Recomendação por nível de dificuldade e afinidade \\
\hline Baghaei et al. [18] & PS & Crianças (9-13 anos) & 12 & Conhecimento em saúde e percepções do sistema \\
\hline Baek et al. [21] & PS & Adultos & 8 & Usabilidade do sistema \\
\hline Pesare et al. [25] & PS & Pacientes e profissionais (hospital) & 41 & Satisfação, motivação e conhecimento \\
\hline Guo et al. [28] & PS & Pacientes diabéticos & 28 & Conhecimento, comportamento e eficácia \\
\hline Gray et al. [32] & PS & Pacientes diabéticos e cuidadores & 13 & Usabilidade e utilidade do sistema \\
\hline Finkelstein et al. [33] & PS & Adultos saudáveis & 9 & Percepções do usuário com o sistema \\
\hline Akker et al. [34] & PS & Pacientes diabéticos & 14 & Conhecimento dos pacientes \\
\hline Orji et al. [35] & PS & Adultos & 6 & Eficácia do protótipo \\
\hline Sultan and Mohan [45] & PS & Membros de uma clínica & 21 & Impressões do protótipo \\
\hline Nordfeldt et al. [48] & PS & Profissionais & 18 & Experiência dos profissionais \\
\hline Joshi et al. [50] & PS & Adultos & - & Aceitabilidade do sistema \\
\hline Kerfoot et al. [5] & $\mathrm{RCT}$ & Pacientes diabéticos & 456 & Controle glicêmico (HbA1c) \\
\hline Boyd et al. [40] & $\mathrm{RCT}$ & Pacientes idosos & 6 & Interação do paciente \\
\hline Abaza et al. [46] & $\mathrm{RCT}$ & Adultos & 73 & Controle glicêmico (HbA1c) \\
\hline Hanberger et al. [51] & $\mathrm{RCT}$ & Crianças/adolescentes diabéticos & 484 & $\begin{array}{l}\text { Frequência de acesso ao portal, páginas visitadas } \\
\text { controle glicêmico (HbA1c) }\end{array}$ \\
\hline Diefenbach et al. [6] & RPS & Pacientes com câncer de próstata & 72 & Aceitabilidade e viabilidade da intervenção \\
\hline Hussain and Athula [23] & PS & Pacientes diabéticos ou não & 10 & Usabilidade e satisfação dos pacientes \\
\hline Saad et al. [43] & PS & Crianças entre 6 e 12 anos & - & Opinião dos usuários e satisfação com o sistema \\
\hline Mendu et al. [13] & PS & Mulheres hispânicas & 66 & Usabilidade e eficácia do sistema \\
\hline Jiménez et al. [27] & PS & Especialistas em computação & 6 & Usabilidade do protótipo \\
\hline Bickmore et al. [37] & $\mathrm{RCT}$ & Pacientes com fibrilação atrial & 120 & Usabilidade do sistema \\
\hline Jacobs et al. [42] & PS & Pacientes com câncer de mama & 12 & $\begin{array}{l}\text { Usabilidade e influência do sistema no uso na } \\
\text { autogestão da saúde }\end{array}$ \\
\hline Boels et al. [19] & $\mathrm{RCT}$ & Pacientes com diabetes & 228 & $\begin{array}{l}\text { Controle glicêmico (HbA1c), status da saúde } \\
\text { satisfação com o uso do sistema }\end{array}$ \\
\hline Smylie et al. [44] & PDS & $\begin{array}{l}\text { Pacientes com doenças } \\
\text { cardiovasculares }\end{array}$ & 47 & $\begin{array}{l}\text { Conhecimento sobre medicamentos e literacia em } \\
\text { saúde }\end{array}$ \\
\hline Wonggom et al. [22] & $\mathrm{RCT}$ & $\begin{array}{l}\text { Pacientes com insuficiência } \\
\text { cardíaca }\end{array}$ & 88 & $\begin{array}{l}\text { Conhecimento sobre insuficiência cardíaca } \\
\text { satisfação com o uso do sistema }\end{array}$ \\
\hline Kumar et al. [1] & PS & Pacientes com diabetes & 146 & Controle glicêmico (HbA1c) \\
\hline Custodio et al. [47] & RCT & Pacientes com diabetes & 110 & Controle glicêmico (HbA1c) \\
\hline
\end{tabular}

A resposta para a quinta questão "Existem sistemas computacionais que aplicam a educação ubíqua no processo de aprendizagem?" deve considerar o artigo de Mendes Neto et al. (2014). Este propôs um modelo que fornece conteúdo personalizado para apoiar a educação ubíqua de acordo com o perfil do usuário. A recomendação de conteúdo ocorre através de algoritmo genético que seleciona conteúdos tais como 
VIII Congresso Brasileiro de Informática na Educação (CBIE 2019)

Anais do XXX Simpósio Brasileiro de Informática na Educação (SBIE 2019)

informações sobre a doença e tratamentos. Nos demais artigos não foi identificado o uso da educação ubíqua na assistência educacional em DCNTs.

Para responder a sexta questão "Existem sistemas computacionais adaptáveis às necessidades do indivíduo (personalização)?" foram identificados 25 artigos (o equivalente a $49,02 \%$ ) que personalizam a solução através dos dados do perfil do indivíduo, tal como uso de medicamentos, atividades físicas, peso corporal, alimentação e dados clínicos.

Por fim a sétima questão "Qual a distribuição dos artigos por base de dados e o local de publicação a cada ano?" é respondida na Figura 3. A maior ocorrência dos artigos selecionados está nas bases de dados IEEE, PubMed, Scopus, ACM e JMIR (11, 10, 10, 9 e 7, respectivamente) e a menor nas bases de dados ScienceDirect, SpringerLink e Wiley (2, 1 e 1, respectivamente). O número de artigos oscilou nos últimos dez anos, com um aumento significativo em 2018 (27,45\%, 14/51). Em termos do local de publicação, cada artigo foi classificado em conferência, workshop e periódico. Sendo assim, considerando o local de publicação, existe uma predominância de artigos publicados em periódicos $(54,90 \%, 28 / 51)$ e conferências $(43,14 \%, 22 / 51)$. Somente um artigo foi publicado em workshop $(1,96 \%, 1 / 51)$.

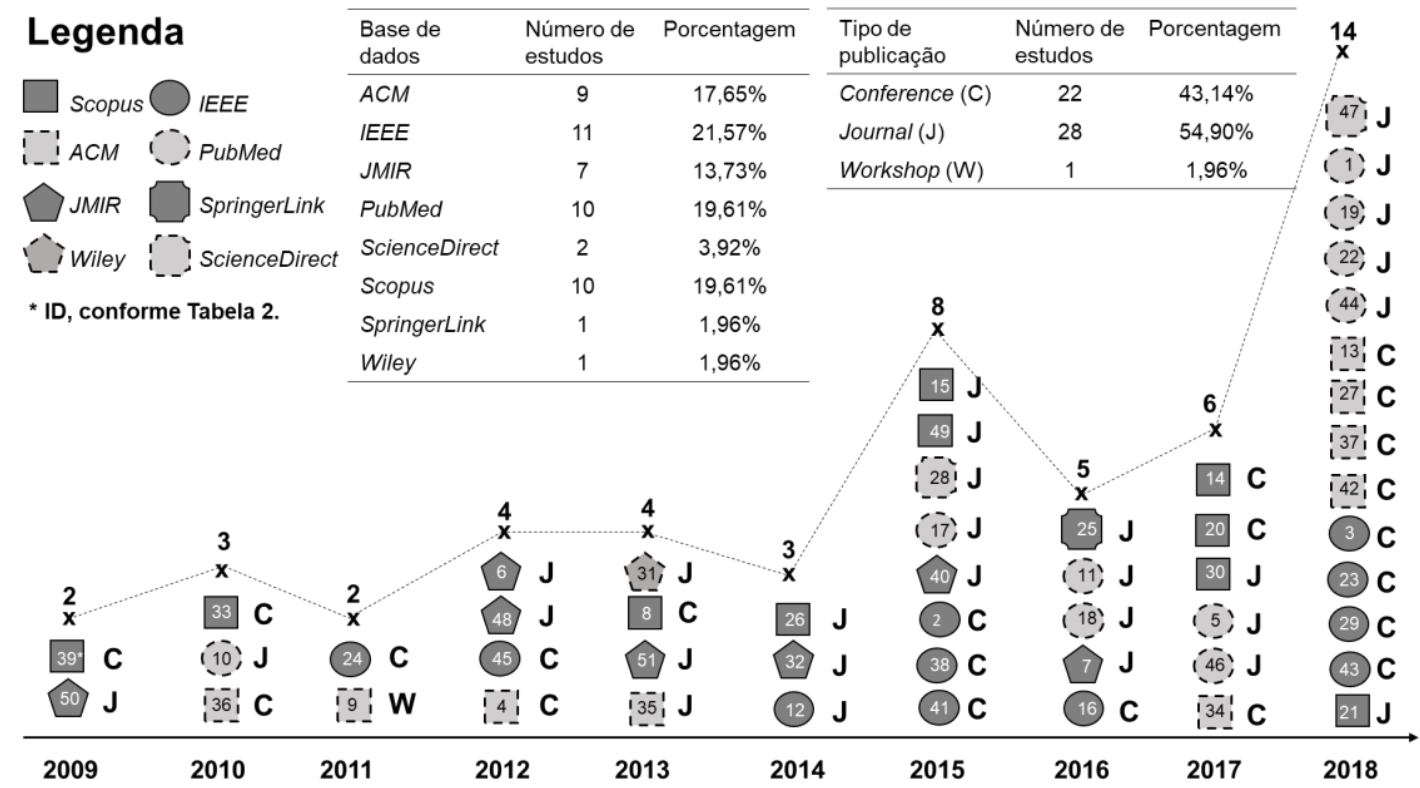

Figura 3. Distribuição dos artigos por base de dados e local de publicação ao longo dos anos (os números nas figuras indicam o ID na Tabela 2).

\section{Considerações Finais}

Este artigo apresenta o resultado de um mapeamento sistemático no qual foram analisados 51 artigos envolvendo o uso da computação na assistência educacional em DCNTs através de diferentes abordagens tecnológicas, também abordando a forma com que as soluções oportunizam o aprendizado aos indivíduos. O estudo identificou que a assistência ocorre por meio da autogestão da doença crônica, uso de jogos educativos e distribuição de conteúdo educacional personalizado. Concluiu-se que diabetes foi a doença crônica priorizada $(60,78 \%, 31 / 51)$ e que cerca de $49,02 \%$ das soluções utiliza dados dos indivíduos para auxiliar em suas condições de saúde. 
VIII Congresso Brasileiro de Informática na Educação (CBIE 2019)

Anais do XXX Simpósio Brasileiro de Informática na Educação (SBIE 2019)

Riscos podem ter impactado nos resultados deste trabalho, por exemplo, a escolha das bases de dados, a seleção dos termos da string de busca, o processo de pesquisa e a definição dos critérios de inclusão e exclusão. Desta forma, para minimizar os riscos, foram selecionadas oito bases de dados reconhecidas no âmbito acadêmico nas áreas de ciência da computação e ciências da saúde. A seleção dos termos da string de busca considerou as áreas da computação, educação e saúde. Na área da computação, termos alternativos para "System" foram utilizados permitindo uma melhor assertividade no retorno da pesquisa. Na educação foi usado o sinônimo "Education" para o termo "Learning" do dicionário online Collins ${ }^{1}$. $\mathrm{Na}$ área da saúde foram consideradas as DCNTs indicadas pela WHO (2018). O processo de pesquisa e os critérios podem ter restringido o acesso de tal forma que artigos relevantes na área de pesquisa podem ter sido removidos. Para mitigar este risco foram utilizadas as técnicas apresentadas por Petersen et al. $(2008,2015)$ e Keshav (2007) as quais também foram aplicadas nos trabalhos dos Bischoff et al. (2019) e Dalmina et al. (2019).

Finalmente, observou-se um amplo uso de aplicações mobile, visto que as soluções podem ser acessadas pelos indivíduos em qualquer lugar e tempo via dispositivos eletrônicos, tais como smartphones e tablets, favorecendo a flexibilidade do aprendizado. Sendo assim, o resultado deste trabalho incentiva pesquisadores da área a explorarem oportunidades referentes ao uso da educação ubíqua na assistência em DCNTs, uma vez que foi encontrado um único artigo com esse foco.

Os seguintes temas destacam-se como tópicos para trabalhos futuros: educação personalizada de acordo com as necessidades do indivíduo, recomendações de conteúdo com base no contexto e recomendação de locais adequados para uma alimentação saudável. As pesquisas futuras podem aplicar a predição de contextos [Rosa et al. 2016] e análise de similaridade de contextos [Wiedemann et al. 2016] para personalização da assistência a DCNTs.

\section{Agradecimentos}

Os autores agradecem à FAPERGS, à CAPES - Código de Financiamento 001, ao CNPq, à Universidade do Vale do Rio dos Sinos (Unisinos), à Universidade Feevale e à Associação de Assistência em Oncopediatria (AMO Criança) pelo apoio ao desenvolvimento desse trabalho.

\section{Referências}

Bischoff, V., Farias, K., Gonçales, L. J., Barbosa, J. L. V. (2019) Integration of feature models: A systematic mapping study. Information and Software Technology, 105:209-225.

Cárdenas-Robledo, L. A., Peña-Ayala, A. (2018) Ubiquitous learning: A systematic review. Telematics and Informatics, 35(5):1097-1132.

Dalmina, L., Barbosa, J. L. V., Vianna, H. (2019) A systematic mapping study of gamification models oriented to motivational characteristics. Behaviour \& Information Technology, pages 1-18.

\footnotetext{
${ }^{1}$ https://www.collinsdictionary.com/dictionary/english-thesaurus/learning
} 
VIII Congresso Brasileiro de Informática na Educação (CBIE 2019)

Anais do XXX Simpósio Brasileiro de Informática na Educação (SBIE 2019)

Heidrich, L., Barbosa, J. L. V., Cambruzzi, W., Rigo, S. J., Martins, M. G., Santos, R. (2018) Diagnosis of Learner Dropout Based on Learning Styles for Online Distance Learning. Telematics and Informatics, 35(6):1593-1606.

Keshav, S. (2007) How to read a paper. ACM SIGCOMM Computer Communication Review, 37(3):83-84.

Mendes Neto, F. M., da Costa, A. A. L., Sombra, E. L., Moreira, J. D. C., Valentim, R. A. M., Samper, J. J., do Nascimento, R. P. C., Flores, C. D. (2014) Content's personalized recommendation for implementing ubiquitous learning in health 2.0. IEEE Latin America Transactions, 12(8):1515-1522.

Petersen, K., Feldt, R., Mujtaba, S., Mattsson, M. (2008) Systematic Mapping Studies in Software Engineering. In 12th International Conference on Evaluation and Assessment in Software Engineering, pages 68-77.

Petersen, K., Vakkalanka, S., Kuzniarz, L. (2015) Guidelines for conducting systematic mapping studies in software engineering: An update. Information and Software Technology, 64:1-18.

Quinde, M., Khan, N., Augusto, J. C. (2018) Personalisation of Context-Aware Solutions Supporting Asthma Management. In 16th International Conference on Computers Helping People with Special Needs, pages 510-519.

Rosa, J. H., Barbosa, J. L. V., Ribeiro, G. D. (2016) ORACON: An Adaptive Model for Context Prediction. Expert Systems with Applications, 45(1):56-70.

Satyanarayanan, M. (2001) Pervasive Computing: Vision and Challenges. IEEE Personal Communications, 4(8):10-17.

Seys, S. F. et al. (2018) mySinusitisCoach: patient empowerment in chronic rhinosinusitis using mobile technology. Rhinology, 56(3): 209-215.

Vianna, H. D., Barbosa, J. L. V., Pittoli, F. (2017) In the Pursuit of Hygge Software. IEEE Software, 34(6):48-52.

Vianna, H. D., Barbosa, J. L. V. (2019) A scalable model for building context-aware applications for noncommunicable diseases prevention. Information Processing Letters, 148:1-6.

WHO (2013) Global Action Plan for the Prevention and Control of noncommunicable diseases 2013-2020. Disponível em:

http://www.who.int/nmh/events/ncd_action_plan/. Acesso em: 4 de Junho de 2019.

WHO (2018) World Health Statistics 2018: Monitoring Health for the SDGs, sustainable development goals. Disponível em: http://www.who.int/gho/publications/world_health_statistics/2018/. Acesso em: 4 de Junho de 2019.

Wiedemann, T., Barbosa, J. L. V., Rigo, S. J., Barbosa, D. N. F. (2016) RecSim: A Model for Learning Objects Recommendation using Similarity of Sessions. Journal of Universal Computer Science, 22(8):1175-1200. 